\title{
Análisis cualitativo de resiliencia en estudiantes de posgrado
}

\author{
A Qualitative Analysis of Resilience in Postgraduate Students
}

\section{Análise qualitativa de resiliência em estudantes de pós-graduação}

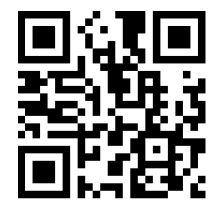

\author{
Lilia Benítez-Corona' \\ Universidad Politécnica de Pachuca \\ lybeco@upp.edu.mx \\ María Concepción Barrón-Tirado² \\ Universidad Nacional Autónoma de México \\ barront@unam.mx
}

\begin{abstract}
Recibido • Received • Recebido: 18 /05 / 2016
Corregido • Revised • Revisado: $03 / 10 / 2017$

Aceptado • Accepted • Aprovado:14/11/2017
\end{abstract}

\begin{abstract}
Resumen: El desarrollo de la resiliencia se adquiere a través de las experiencias de vida del individuo. Desde esa tesitura, se presentan resultados obtenidos en la investigación posdoctoral: “El desarrollo de competencias resilientes en el estudiantado del posgrado en Pedagogía de la Universidad Nacional Autónoma de México (UNAM). Resiliencia es la capacidad de vivir de forma positiva a pesar de la adversidad o situaciones dificiles que enfrentan las personas en los diferentes contextos sociales. Dada su subjetividad, su análisis es complejo, pues las dificultades son percibidas de forma diferente en cada individuo. El objetivo del artículo es mostrar el análisis de la resiliencia desde la mirada del estudiantado de posgrado para identificar qué factores han desarrollado su resiliencia, cómo la construyeron y de qué forma ha impactado en su formación como especialistas en investigación educativa. El acercamiento metodológico cualitativo fue a través de la hermeneútica para reconocer la diversidad, comprender la realidad y construir sentido a partir de la comprensión histórica del mundo simbólico del individuo, ya que la conducta humana solo se puede comprender a través de los significados. Por lo mismo, se empleó la técnica del taller, cuyo objetivo fue que los sujetos participantes descubrieran su resiliencia. La muestra se constituyo de 23 estudiantes de posgrado. Los hallazgos mostraron que la interacción positiva, el sentido de vida yla presencia del otro significativo fomentaron esta capacidad en algunos estudiantes al favorecer el pensamiento crítico y la autoestima consciente, factores protectores necesarios en la formación de personal investigador educativo.
\end{abstract}

Palabras claves: Estudiantes de posgrado; interacción positiva; resiliencia; sentido de vida.

\footnotetext{
${ }^{1}$ Doctora en Ciencias de la Educación. Profesora-investigadora de la Universidad Politécnica de Pachuca. Miembro del Sistema Nacional de Investigadores nivel I. Áreas de trabajo: resiliencia, educación superior y aprendizaje.

${ }^{2}$ Doctora en Pedagogía. Profesora-investigadora del IISUE y directora de Desarrollo Educativo en el CUAD, UNAM. Miembro del Sistema Nacional de Investigadores nivel II. Áreas de trabajo: currículo, formación y vinculación.
} 
doi: http://dx.doi.org/10.15359/ree.22-1.7

URL: http://www.una.ac.cr/educare

CORREO: educare@una.cr

\begin{abstract}
The development of resilience is acquired through the life experiences of individuals. In this way, the results obtained in postdoctoral research are presented: "The development of resilient competences in graduate students in Pedagogy of the National Autonomous University of Mexico (UNAM)". Resilience is the ability to live positively despite the adversity or difficult situations faced by people in different social contexts. Given its subjectivity, analyzing turns complex, because difficulties are perceived differently by each individual. The aim of the article is to show the analysis of resilience from the point of view of postgraduate students in order to identify what factors have developed their resilience, how they built it and how it has impacted their training as specialists in educational research. The qualitative methodology was approached through hermeneutics to recognize diversity, understand reality and build meaning from the historical understanding of the symbolic world of the individual, since human behavior can only be understood through meanings. For this reason, the workshop technique was used to let the participants to discover their resilience. The sample consisted of 23 graduate students. The findings showed that the positive interaction, the sense of life and the presence of the significant other encouraged this capacity in some students by favoring critical thinking and conscious self-esteem, necessary protective factors in the training of educational researchers.
\end{abstract}

Keywords: Resilience; postgraduate students; sense of life; positive interaction.

Resumo: O desenvolvimento da resiliência é adquirido através das experiências de vida do indivíduo. Nesta perspectiva, são apresentados os resultados obtidos na pesquisa pós-doutoral "O desenvolvimento de competências resilientes nos alunos do curso de pós-graduação em Pedagogia da Universidade Nacional Autônoma do México (UNAM)". Resiliência é a capacidade de viver de maneira positiva apesar da adversidade ou dificuldade enfrentada pelas pessoas em diferentes contextos sociais. Dada a sua subjetividade, sua análise é complexa, porque as dificuldades são percebidas de forma diferente em cada indivíduo. O objetivo do artigo é mostrar a análise de resiliência a partir da perspectiva dos estudantes de pós-graduação para identificar quais fatores desenvolveram sua resiliência, como eles o construíram e como isso impactou sua formação como especialistas em pesquisa educacional. A abordagem metodológica qualitativa foi através da hermenêutica para reconhecer a diversidade, compreender a realidade e construir o significado a partir da compreensão histórica do mundo simbólico do indivíduo, desde que o comportamento humano só pode ser entendido através dos significados. Portanto, utilizou-se a técnica da oficina, cujo objetivo era que os participantes descobrissem sua resiliência. A amostra foi constituída por 23 estudantes de pós-graduação. Os resultados mostraram que a interação positiva, o senso de vida e a presença do outro significado encorajaram essa habilidade em alguns estudantes ao favorecer o pensamento crítico e a autoestima consciente, fatores de proteção necessários ao treinamento de pessoal de pesquisa educacional.

Palavras-chave: estudantes de pós-graduação; interação positiva; resiliência; sentido da vida.

\title{
La resiliencia en el desarrollo humano
}

Las investigaciones sobre resiliencia en los seres humanos se han llevado a cabo desde el siglo pasado y muestran que las personas siempre han tenido la capacidad de superar obstáculos. “Esa capacidad ahora se conoce como resiliencia” (Manciaux, 2002, p. 12). 
Crisis económicas, desastres naturales, adicciones, marginación, desigualdad, guerras, hambre, pobreza, enfermedades, familias en conflicto, personas con capacidades diferentes, infancia en abandono, madres y padres solteros, violencia y demás situaciones que ponen en riesgo la integridad del individuo causan en él una fuerza que no solo es un rebote a la adversidad, sino también una verdadera voluntad para salir adelante y buscar una calidad de vida más alta. En palabras de Werner (1989), se diría que son personas que han desarrollado su capacidad resiliente.

El avance de los estudios sobre resiliencia permite identificar a dos pioneros del concepto: Werner (1989) y Rutter (1985). La primera refiere su análisis desde un enfoque conductista, prágmatico y centrado en lo individual; identifica la resiliencia con la capacidad de enfrentar adversidades y de autocontrolar sus emociones, lo que evita la reactividad y genera respuestas proactivas, lo que se considera la libertad de elegir y dar un sentido positivo a la situación. Rutter (1985), en cambio, señala evidencias de resiliencia en términos del análisis de la interacción recurrente entre las personas y el medio ambiente; además destacó el papel activo de los individuos frente a lo que les ocurre.

A partir de sus aportaciones, surgen múltiples trabajos a nivel internacional en torno a la resiliencia. Ejemplo de ello es la resiliencia comunitaria, aporte latinoamericano, porque se aplica especialmente en la investigación de grupos para analizar la capacidad colectiva frente a las adversidades (Suárez, De la Fara y Márquez, 2007), dadas las situaciones de pobreza extrema, discriminación y desigualdad.

En este sentido, Melillo (2006, p. 72), señala que "la resiliencia se teje: no hay que buscarla solo en la interioridad de la persona, ni tampoco en su entorno, sino en ambos, pues es un proceso íntimo con el entorno social". Por ello, la resiliencia se conceptualiza como un proceso en que los mecanismos internos del ser humano, al entrar en contacto con su entorno familiar y social, potencializan los aspectos positivos.

Por otra parte, Grotberg (1996) discurre que, para hacer frente a las adversidades, la niñez toma elementos de resiliencia de cuatro fuentes interactivas que se visibilizan en las expresiones verbales de niños, niñas, adolescentes y personas adultas, como "yo tengo", en el entorno social; "yo soy" y "yo estoy", que hablan de las fortalezas intrapsíquicas y condiciones personales; "yo puedo", que se vinculan con las habilidades en las relaciones con los otros individuos. De esta manera, Grotberg define el concepto resiliencia como la capacidad del ser humano para hacer frente a las adversidades de la vida, superarlas e incluso transformarse a causa de ellas.

En consecuencia, para efectos de esta investigación, se define resiliencia como la competencia del ser humano para hacer frente a los problemas, solucionarlos y salir fortalecido al enfrentarlos; es decir, que, en la circunstancia de sobrellevar la adversidad, el individuo sufre una transformación interna que le da más seguridad en la toma de decisiones benéficas para su vida. 
doi: http://dx.doi.org/10.15359/ree.22-1.7

URL: http://www.una.ac.cr/educare

CORREO: educare@una.cr

\section{Resiliencia en el posgrado}

Las etapas de vida tienen elementos que forman personalidad, carácter, pensamientos y estilos. La historia individual de la persona adulta está marcada por acontecimientos familiares e interacción con otras personas: estilos de vida, formación y separación de parejas, nacimientos, enfermedades, muertes, migraciones, entre otras; en un tiempo histórico y social cambiante. Poco a poco el individuo se transforma, adquiere nuevas ideas y edifica su historia.

Al respecto, Ausubel (1954) señaló que, mientras en la niñez la dependencia deriva de las condiciones biológicas, en la juventud, la dependencia deriva de factores sociales, culturales y económicos. Por lo mismo, surge la necesidad de mejorar su calidad de vida tanto a nivel intelectual como emocional.

La adolescencia se alimenta de vínculos afectivos que no solo protegen al sujeto, sino que además le ayudan a desarrollar sus emociones: le permiten reconocer sus limitaciones, descubrir todo aquello de lo que es capaz.

En consecuencia, si a lo largo de su vida el sujeto ha recibido atenciones, en la etapa adulta asume conductas positivas para ayudar a otros sujetos, porque en la niñez entendió que en un momento dado se puede depender de otros (Rubio y Puig, 2015).

Por ello, las personas adultas, al enfrentar cambios en las organizaciones y en la división del trabajo generados por el avance de la ciencia y tecnología, regularmente optan por estudiar una carrera universitaria. Sin embargo, el mundo globalizado demanda una mayor preparación que se consigue en el posgrado.

En México, actualmente, existen 8,522 programas de posgrado en 1,723 instituciones. De ellas, 1,134 son particulares y 289, públicas. Se distribuyen en 4,230 programas de maestría, 1,849 de especialidad y 890 de doctorado. Están incorporados al Programa Nacional de Posgrado de Calidad (PNPC) 1691. El 58,8\% corresponde a programas de doctorado: 132 en ciencias básicas, 208 en ciencias aplicadas y 184 en humanidades y ciencias sociales.

Las estadísticas en 2011 identificaban cerca de 251 mil estudiantes de posgrado; es decir, alrededor 76 por cada mil en educación superior. La mayoría realizaba estudios de maestría (72 de cada 100) y solo una minoría (11 de cada 1000) cursaba un doctorado (Tuirán, 2012).

En el caso de la Universidad Nacional Autónoma de México, se ofertan 130 programas de posgrado a nivel maestría, doctorado y especialización que abarcan áreas del conocimiento en ciencias físico-matemáticas y de las ingenierías, ciencias biológicas, químicas y de la salud, ciencias sociales, humanidades y de las artes. En estos programas, se atendió hasta el periodo 2012-2013 a una población de 27.058 estudiantes, lo cual representó el 11.69\% de la población total a nivel nacional. 
Dentro de los posgrados se encuentra el de Pedagogía, que tiene los grados de maestría y doctorado. En 2014, atendió a una población de 373 estudiantes en ambos programas.

Ingresar a un posgrado implica tomar una decisión ligada al plan de vida del individuo. La población del Posgrado en Pedagogía se ubica en la edad adulta, que, según Erikson (1998), se identifica como una de las etapas más largas de la vida del ser humano. El autor considera que este periodo trascurre de los treinta a los sesenta años. Además, distingue dos periodos: primera edad adulta (de treinta a cincuenta) y segunda edad adulta (desde los cincuenta a los sesenta), ya que los intereses y preocupaciones de una persona de treinta no son los mismos que los de una de sesenta.

Los esquemas de vida adquieren una mirada diferente que va cambiando de manera radical a partir de los treinta años. Al llegar a los sesenta, el individuo ha acumulado experiencias de vida que combinan con frecuencia componentes como reinicios, participación en alguna actividad, la responsabilidad de algo o de alguien, la esperanza. En el fondo, la existencia de un vínculo positivo entre la vida del sujeto y la mayor corriente social fluye junto a él y genera el sentimiento que le da sentido a su vida (Vanistendael, 2006).

El posgrado es un nivel educativo inalcanzable para un alto porcentaje de la población en México. Laclette (2014) señaló que únicamente dieciséis de cada diez mil habitantes estudian algún posgrado. Hay que considerar también que permanecer en un posgrado es difícil, ya que la formación en investigación implica desarrollar capacidades de análisis, síntesis, criticidad, creatividad, entre otras; además requiere esfuerzo, disciplina y responsabilidad para enfrentar las exigencias académicas que demanda.

Lo anterior justifica que un criterio relevante para ingresar al posgrado sea la trayectoria académica. Este concepto se refiere al "comportamiento académico de un individuo e incluye el desempeño escolar, la aprobación, la reprobación, el promedio logrado... a lo largo de los ciclos escolares" (García y Barrón, 2011, p. 95). Analizar la trayectoria académica proyecta la posibilidad del estudiantado para permanecer y concluir su posgrado. Sin embargo, también es importante considerar las experiencias de vida de las personas candidatas, porque pueden dar cuenta de sus fortalezas y debilidades.

Al respecto, Téllez (citado por Martínez, 2013) afirmó que el fracaso en el posgrado se da porque se estudia solo para escalar a una posición de mayor rango. No se visualiza que la mejoría tanto en el plano económico como en el personal es producto de la convicción y la pasión por lo que se hace. Estudiar un posgrado nace de la creencia y de la vocación por ser un mejor individuo; no necesariamente como un mecanismo para obtener un mejor salario. Lo prioritario es elevar la calidad de la vida humana. El desarrollo económico viene por añadidura. 
doi: http://dx.doi.org/10.15359/ree.22-1.7

URL: http://www.una.ac.cr/educare

CORREO: educare@una.cr

Por otra parte, Sánchez (2006, citado en García y Barrón, 2011) menciona que hay factores -la tutoría durante la tesis, los conocimientos adquiridos en su formación, la falta de prácticas de investigación y el poco interés del alumnado, entre otros- que impactan el índice de graduación de estudiantes de posgrado de Pedagogía. En este sentido, Torres-Frías (2012) afirma que en el programa de doctorado dentro de la "tutoría y los coloquios de investigación... se hace evidente la presencia de algunas manifestaciones de desigualdad educativa, jerarquías de poder y violencia simbólica" (p. 115), situaciones que surgen a lo largo de los procesos de formación en el doctorado. Estas se convierten en una fuente de reflexiones múltiples sobre las condiciones académicas en que el estudiantado de doctorado vive la formación para la investigación y su transformación progresiva o conversión como sujeto investigador educativo.

El testimonio anterior ofrece evidencias de las situaciones que estudiantado maestrante y doctorando enfrenta, y que ponen en riesgo su formación en investigación educativa. No obstante, al mismo tiempo, estas circunstancias pueden ser un impulso para comprometerse consigo mismos y alcanzar sus objetivos. Esta situación permite observar la dualidad de los factores que Rutter y Rutter (1992) señalaron como factores protectores y factores de riesgo.

Ahora bien, es importante considerar que tanto los factores de protección como los de riesgo promueven en el individuo la construcción y el desarrollo de la resiliencia. En este sentido, desde la teoría sistémica, que se sitúa en un nivel transdisciplinario, es posible concebir al mismo tiempo no solo la naturaleza material del objeto, sino también los tipos y complejidades de los fénomenos asociados. Por ello, su campo de acción se extiende a lo cognoscible (Morin, 2005).

Por lo anterior, el riesgo puede convertirse en factor de protección, y un factor de protección, en uno de riesgo. ¿De qué depende? Del sujeto, de la situación que viva y del lugar donde ocurra; por eso se afirma que la resiliencia es subjetiva.

La palabra resiliencia surge en la física y se refiere a la capacidad de los cuerpos para regresar a su forma original luego de haberse deformado por la fuerza aplicada en ellos (Suárez, 2004). Viene del término latino resilio, que significa volver atrás, volver en un salto, rebotar. En las ciencias sociales, se ha identificado con casos de personas que, a pesar de enfrentar condiciones difíciles, superan obstáculos, resisten, continúan, lo que hace de ellas personas integradas socialmente, mientras que otras en las mismas situaciones dejan de esforzarse.

Algunos trabajos de resiliencia realizados en Latinoamérica muestran hallazgos importantes referidos a situaciones de pobreza, salud y desastres naturales. Han sido abordados desde un enfoque social, por lo que se consideran los contextos donde se desarrolla el individuo y se investiga a través de los ambientes familiares, escolares, comunitarios, etc. Por lo mismo, Suárez y Krauskopf (1995) entienden la resiliencia como una combinación de factores que le permiten al individuo afrontar y superar los problemas y adversidades de la vida. 
Pero, si la resiliencia es la capacidad del ser humano para enfrentar circunstancias adversas, ¿de dónde proviene?, ¿solo algunos la poseen? Según Grotberg (2006, p. 20) "todos pueden volverse resilientes. El desafío es encontrar la forma de promoverla en cada individuo".

En las primeras investigaciones sobre resiliencia, realizadas por Werner y Smith (1982), Garmezy (1991) y Rutter (1979), se enumeraron factores que contribuían a identificar lo útil y afectivo a la hora de superar adversidades; los denominaron factores protectores. Este concepto alude a las influencias que modifican, mejoran o alteran la respuesta de una persona a algún peligro que predispone a un resultado no adaptativo (Rutter, 1985). Es entonces cuando, en situaciones adversas, la respuesta resiliente de las personas está conformada por los factores protectores que se distinguen de las experiencias positivas en que aquellos incluyen un componente de interacción. Las experiencias positivas dan resultados adaptativos; los factores protectores, por su parte, manifiestan sus efectos ante la adversidad, modificando la respuesta del sujeto en un sentido comparativamente más adaptativo que el esperable. Es así como un factor protector puede no constituir una experiencia en absoluto, sino una cualidad o característica individual de la persona (Rutter, 1979).

Por lo anterior, a partir de las contribuciones, Melillo (2004) identifica los protectores y los denomina "pilares de la resiliencia", a saber:

- Autoestima consciente. Es parte de las vivencias que se desarrollan en la niñez, de los cuidados que le fueron otorgados y que en la edad adulta emergen cuando se observa cómo se relaciona con los demás seres. El sujeto se siente seguro de sí mismo en cualquier contexto.

- Introspección. Es la capacidad de comunicación del individuo consigo mismo. Se representa cuando el sujeto es capaz de identificar sus estados de ánimo.

- Creatividad. Inicia con la imaginación, a través de ideas, de pensamientos que le dan al individuo la capacidad de innovación, de crear, de mejorar.

- Iniciativa. Es la capacidad que desarrolla el ser humano en la toma de decisiones.

- Pensamiento crítico. Permite el análisis de las causas de la adversidad para concientizar la forma en que se toman decisiones.

- Independencia. Es la capacidad del ser humano de establecer límites consigo mismo y con el contexto que le rodea. Implica mantener distancia ante situaciones adversas sin caer en la reclusión.

- Capacidad de relacionarse. Identificada como la capacidad humana de establecer relaciones sociales, de crear lazos, de mantenerse en contacto, de recibir reconocimiento para mantener en equilibrio la necesidad de pertenencia. 
doi: http://dx.doi.org/10.15359/ree.22-1.7

URL: http://www.una.ac.cr/educare

CORREO: educare@una.cr

- Humor. Encontrar una sonrisa en la tristeza, rescatar de las pérdidas ganancias; es decir, desarrollar la capacidad de mirar la parte positiva de la adversidad.

- Moralidad. La capacidad de comprometerse con los valores, teniendo en cuenta que en cada contexto se mira diferente la adversidad y lo que es bueno para unas personas no es bueno para otras.

Por lo que, al analizar la mirada del estudiantado del posgrado en Pedagogía, se observa que, a pesar de las situaciones difíciles durante su formación como personal investigador educativo, una parte las enfrentan, las resuelven y cambian su perspectiva ante el problema.

En consecuencia, a partir de las consideraciones anteriores, la investigación tuvo como objetivo general interpretar, a través de la mirada de la comunidad estudiantil del posgrado en Pedagogía, los factores que construyeron su resiliencia y cómo fortalecieron su formación como sujetos investigadores educativos.

En resiliencia los factores pueden tener las perspectivas de protección y riesgo. Estas se consideraron como ejes de análisis en este trabajo.

\section{Acercamiento metodológico}

Las particularidades de la metodología cualitativa de Taylor y Bogdan (2013) la caracterizan como inductiva, holística, sensible a los efectos que causa sobre las personas que son objeto de estudio. Esto permite inquirir sobre los procesos de investigación social en el momento en que se presentan; también facilita la construcción y reconstrucción del modelo que se estudia.

Mella (1998) señala que una característica fundamental en la investigación cualitativa es su claro planteamiento de comprender los acontecimientos, acciones, normas y valores, por mencionar algunos. Apunta que la estrategia de tomar esta perspectiva se presenta "generalmente en términos de 'ver a través de los ojos de la gente que uno está estudiando'" ( $\mathrm{p}$. 8), por lo que es necesario empatizar quienes están siendo objeto de estudio.

El planteamiento anterior justifica la elección del enfoque hermenéutico que, de acuerdo con las aportaciones de Vélez y Galeano (2000, p. 89), se considera como una "herramienta que busca reconocer la diversidad, comprender la realidad y construir sentido a partir de la comprensión histórica del mundo simbólico". La conducta humana, según Mead (1972), solo puede comprenderse en relación con los significados, ya que le permiten al individuo orientarse para determinar la dirección en que están sus objetivos. También, no obstante, pueden obstaculizar su paso. Lo anterior depende de su mirada resiliente que, en esta investigación, repetimos, se considera como la capacidad de enfrentar, resolver y cambiar su perspectiva ante el problema.

8 Lilia Benítez-Corona y María Concepción Barrón-Tirado

Los artículos de la Revista Electrónica Educare del Centro de Investigación y Docencia en Educación de la Universidad Nacional, Costa Rica, se comparten bajo términos de la Licencia Creative Commons: Reconocimiento, № Comercial, Sin Obra Derivada 3.0 Costa Rica. Las autorizaciones adicionales a las aquí delimitadas se pueden obtener en el correo: educare@una.cr 
La resiliencia se ha planteado tanto en metodologías cuantitativas como cualitativas. Las de mayor profundidad y cercanas al sujeto se ubican en las cualitativas. Al respecto, Suárez (2004) señala que las investigaciones de resiliencia se han elaborado a través de marcos referenciales interpretativos circunscritos en enfoques etnográficos, análisis convencionales, hermenéutica y fenomenología.

Por lo mismo, se abordó el análisis desde la perspectiva de la hermenéutica y se utilizaron las historias de vida como modalidad de la investigación cuyo objetivo es el estudio de la persona; donde el sujeto es el centro de conocimiento y reconocimiento de si mismo en su dinámica y trayectoria, en tiempos y espacios, y en su pertenencia a una cultura en particular (Godard y Cabanes, 1996).

Las aportaciones de Cyrulnik (2010) destacan que todas las personas que han sido heridas durante la infancia se asombran, al llegar a la edad adulta, del número de manos tendidas que lograron encontrar.

El concepto de resiliencia, por lo tanto, debe entenderse como un proceso que se construye en lo social, lo relacional y los ecosistemas humanos. En consecuencia, estos procesos de construcción social de la resiliencia están, a su vez, mediados por las trayectorias históricas y contenidos culturales propios del contexto que van a condicionar los significados individuales y colectivos (Madariaga, Palma, Surjo, Villalba y Arribillaga, 2014).

Desafortunadamente, en la actualidad se viven escenarios de violencia e inseguridad a los que las personas ya se han acostumbrado. Es habitual que exista la prepotencia, la discriminación y los malos tratos en la mayoría de los contextos sociales. De ahí que el ser humano se encuentra vulnerable, de modo que urge la necesidad de cambiar la mirada hacia escenarios diferentes donde las personas se reconozcan y se traten como personas. Se necesitan escenarios en donde la persona se valore como tal.

Es por ello que se busca, desde la mirada de los sujetos integrantes del posgrado, qué factores en su historia de vida incidieron en la construcción y desarrollo de su resiliencia, los cuales emergen de la dimensión ética del individuo, porque se manifiestan en función de sus propios valores, percepciones y objetivos.

Rousseau (2012), en concordancia con lo anteriormente mencionado, señala que el ser humano se apoya en sus valores cuando toma decisiones, y que estos caminan con el individuo en los malos y buenos momentos, sobre todo cuando atraviesa una tormenta emocional acompañada de factores de riesgo que pueden transformase en factores de protección y viceversa. Estos pueden constituirse en la puerta de entrada para desarrollar la resiliencia.

Sin embargo, esta relación entre los factores de protección y de riesgo se torna compleja y a veces confusa. Por ello, la dimensión ética está fuertemente ligada a la resiliencia (Vanistendael, 
doi: http://dx.doi.org/10.15359/ree.22-1.7

URL: http://www.una.ac.cr/educare

CORREO: educare@una.cr

2014). Los factores protectores como la autoestima, pensamiento crítico, la búsqueda de sentido, entre otros, deben considerarse desde un punto ético. Por ejemplo:

La autoestima, mientras sea moderada y no se trasforme en egocentrismo, prepotencia o arrogancia, se puede considerar como un factor protector, que se manifiesta con la seguridad en sí mismo y al mismo tiempo crea confianza en el contexto, pues desarrolla comprensión y empatía con los otros.

El pensamiento crítico, que permite analizar, comprender y evaluar la realidad y las complejas situaciones que se nos presentan.

La búsqueda de sentido, que se refiere a la capacidad de encontrarle un sentido a la vida o a las heridas en función de nuestra historia, de nuestros valores y nuestras creencias (Cyrulnik, 2006).

Así, en las historias de vida de 23 estudiantes del posgrado en Pedagogía de la UNAM se identifican elementos de resiliencia, como la interacción positiva, el sentido de vida y la presencia del otro significativo, presentes en la historia del individuo.

\section{Procedimiento}

De acuerdo con Cifuentes (2011), una vez que se tiene el enfoque cualitativo -en este caso, la hermenéutica y la historia de vida como modalidad de investigación-, un tercer elemento que complementa este proceso es la técnica, canal que da la pauta para obtener información.

Una de las técnicas utilizadas en la investigación cualitativa son los talleres. Ghiso (1999, p. 142) entiende el taller como un "instrumento ... para la apropiación y desarrollo de conocimientos, actitudes y competencias de manera participativa y pertinente a las necesidades [de una investigación]".

Dado lo anterior, se diseñó e impartió el taller "Innovación cognitiva para descubrir la resiliencia", cuyo objetivo fue que la persona participante descubriera su resiliencia para elevar su calidad de vida, mejorar sus ambientes de trabajo e incrementar su comunicación asertiva.

El taller se impartió en cuatro sesiones de cuatro horas cada una (16 en total). Los temas fueron: I. Resiliencia y adversidad, II. Fuentes y hábitos del pensamiento, III. Valores y comunicación, y IV. Adaptación positiva.

Se ofertó a través de una convocatoria, por lo que se trabajó con dos grupos del Posgrado en Pedagogía: el primer grupo con 14 estudiantes y el otro, con 9. Por lo tanto, la muestra fue de 23 estudiantes: 18 de maestría y 5 de doctorado. 
La secuencia para que cada estudiante escribiera su historia de vida fue la siguiente:

1) En la sesión I. Resiliencia y adversidad, se presentaron diapositivas la vida del Premio Nobel de Genética Molecular Mario Capecchi para inducir al estudiantado de posgrado a reflexionar sobre su historia de vida.

2) Proporcionar una guía para orientar el análisis de su historia de vida. Esta "es la hebra analítica que une e integra los principales... datos" (Taylor y Bogdan, 2013, p. 166).

3) Para analizar las historias de vida, se consideraron tres categorías: identificación de problemas y solución, el otro sujeto significativo o tutor resiliente y sentido de vida.

\section{Resultados: Miradas resilientes (MR)}

Al considerarse la familia como la base de la sociedad, en donde se ama, se cuida y se educa a los otros individuos, se espera que forme ciudadanos y ciudadanas con valores y creencias basadas en el bienestar colectivo. Sin embargo, un análisis desde la mirada resiliente del estudiantado de posgrado identificó que la mayoría vivió problemas en su infancia, como discriminación, infidelidad, abandono, violencia y alcoholismo. En este sentido, la funcionabilidad o disfuncionalidad de la familia no depende de la ausencia de problemas; por el contrario, depende de la respuesta que muestre frente a estos (Minuchin y Fishman, 1984).

En este artículo, los problemas se conceptualizan como adversidades; de acuerdo con Lemaître y Puig (2005), estas pueden considerarse como la exposición de una persona a un conjunto de riesgos de diferentes tipos.

A continuación, se presentan los testimonios que dan cuenta de algunas de las adversidades presentadas en la niñez del estudiantado de posgrado, que, al enfrentarlas, favorecieron su resiliencia.

\section{Identificación de problemas y solución}

Diversas situaciones generan en la mayoría de las personas traumas. Cyrulnik (2005, p. 24) señala que "no se puede hablar de un proceso de resiliencia sin existir un trauma. La diferencia entre trauma y prueba radica en que en esta última se lucha, se sufre, hay depresión o enojo, el individuo sigue siendo él y acaba superándola".

Al respecto, Puig y Rubio (2011, p. 173) plantearon que "el trauma es algo imprevisible, que hay que sobrevivir, y que provoca una pérdida de una parte de sí mismo... En la prueba en cambio, la persona permanece íntegra, angustiada... pero definida con lo que es, con lo que funciona bien en ella". 
doi: http://dx.doi.org/10.15359/ree.22-1.7

URL: http://www.una.ac.cr/educare

CORREO: educare@una.cr

Por lo tanto, la tragedia del trauma se transforma en energía creadora y enriquece la vida de la persona. Aceptarla y sobreponerse a ella es algo positivo. En las personas resilientes, una vez superado el trauma, se genera un sentimiento común: aumento de la confianza en sí mismas para afrontar las adversidades del futuro.

En consecuencia, en algunos testimonios se identifican experiencias que dan cuenta de la transformación de pensamiento que vivió la persona ante una adversidad:

Mi situación económica cuando era niña era muy baja, asistí a una escuela pública, pero había muchos niños ricos que se compraban comida en el recreo y tomaban refresco, se reían de mí porque siempre llevaba mi torta de frijoles. He logrado transformar esa experiencia negativa en algo hermoso... mi mamá siempre me amó porque nunca dejó de prepararme mi torta para la escuela. (MR17)

Mi mamá era enfermera y murió cuando yo tenía 2 años y medio por una enfermedad que aparentemente adquirió en el hospital en donde trabajaba. Como mi papá tenía otra mujer (y para entonces otro hijo con ella), mis abuelos paternos nos recogieron, pero a mi hermano se lo llevo una tía y yo me quede con mi hermana mayor, ella cuidó de mí y con su amor me impulsó para seguir adelante. (MR2)

Otra forma en que las personas aprenden a socializar es a través de la escuela, que, además de presentar retos académicos, también tiene retos sociales para el sujeto, porque debe aprender a convivir con los demás seres (Delors, 1992).

Un problema importante que se ha identificado en la niñez se refiere a la apariencia física. Este se traduce en etiquetación en la mayoría de los contextos sociales, como indica Watzlawick (1976). La etiquetación influye tanto en la persona como en todo el resto, actúa como profecía de la autorrealización personal, que por el solo hecho de haber sido pronunciada, logra desencadenar muchas situaciones en que el sujeto es vulnerable (Fiorenza, 2009).

En consecuencia, el uso de etiquetas se presenta con una doble intención: la agresiva de corte peyorativo y la positiva que da reconocimiento, como puede advertirse en los siguientes casos:

Nací en 1981, mis padres, dos indígenas de Oaxaca que nos trajeron al mundo en la pobreza. No es mi primer recuerdo, pero sí algo que me marcó en la niñez: como en cualquier otro hijo de campesinos, el tener que trabajar en la tierra y al mismo tiempo asistir a la escuela. (MR 2)

En la primaria un aspecto que me puso triste fue cuando una niña me dijo burra, eso hizo que la escuela me empezará a importar y cambié, empecé a estudiar y mejoré mis calificaciones. (MR7) 
El uso de etiquetas influye tanto en la persona etiquetada como en todas las demás, en las miradas de resilientes la solución fue trasformar la etiqueta en algo positivo que impulsó su recorrido hacia sus metas personales que dieron sentido a su vida.

El sentido de vida brota al descubrir el reconocimiento que otra gente le da a la persona. Por lo mismo, en la mayoría de las personas adultas, encontramos heridas que, a través del desarrollo de su resiliencia, se rehabilitan y hacen de su vida una obra maestra (Cyrulnik, 2010), como es el caso de algunos o algunas estudiantes del Posgrado en Pedagogía. Vanistendael y Lecomte $(2002$, p. 69) señalan que "el sentido de vida va más allá de una reflexión intelectual o vivir sin objetivos. El sentido de vida devuelve al individuo la capacidad de proyectarse, de ser mejor como persona".

Es evidente que, gracias a su mirada resiliente, el estudiantado de posgrado identificó factores de riesgo que, en vez de derrotarlo, lo impulsó a continuar en la búsqueda de sentido, ya que los transformó en áreas de oportunidad en lugar de verlos como derrotas.

\section{El otro sujeto significativo o personal tutor resiliente}

La mirada resiliente del estudiantado de posgrado se construyó también por influencia de los otros sujetos: la presencia de alguien que le valoró, amó y aceptó incondicionalmente. Rutter (1985) señaló que factor protector son las influencias que modifican, mejoran o alternan la respuesta de una persona a algún peligro que predispone a un resultado no adaptativo.

En la interacción positiva emerge el otro sujeto significativo o personal tutor resiliente, la persona que acompaña al individuo herido de manera incondicional, convirtiéndose en su sostén al mismo tiempo que lo impulsa a continuar su trayectoria de vida (Cyrulnik, 2008).

La representación del personal tutor resiliente también puede ser una actividad, un interés e incluso algo más efímero como un personaje, una canción o una película (Puig y Rubio, 2011), "o a veces el amor es un ser humano, otras veces es el mar y, en tal caso, uno se hace buzo o surfista; otras un instrumento musical... ese impulso que nos lleva hacia el otro es fuente de vida. La vida sin el otro no puede vivirse" (Cyrulnik, 2008).

En la mirada resiliente del estudiantado de posgrado, se encontraron evidencias del otro significativo o personal tutor resiliente en la figura de docentes, abuelos y abuelas, hermanos y hermanas:

Éramos muy pobres, mi abuelo era pensionado y cuando yo tenía 8 años mi abuelita murió, recuerdo que yo andaba siempre en la calle jugando con los zapatos rotos, pero me gustaba mucho ir a la escuela, aprendí a leer primero que todos mis primos de mi edad y era la consentida de mis profesores, mis primos mayores de alguna manera nos querían y todos los vecinos nos compadecían por ser huérfanos. (MR 9) 
doi: http://dx.doi.org/10.15359/ree.22-1.7

URL: http://www.una.ac.cr/educare

CORREO: educare@una.cr

Tengo una abuelita, mamá de mi papá que me cuidó y consintió siempre y hasta la fecha lo hace. Fue quien me protegió de los problemas de mis padres y me aceptó tal como era. (MR10)

Las relaciones en la familia y en la escuela son el origen de las conductas del ser humano. La empatía es un elemento que permite identificar los estados de ánimo de los otros sujetos y lograr con esto la comprensión de sus actitudes. La presencia del otro ser, entonces, es fundamental.

Las cualidades del personal tutor resiliente señaladas por Puig y Rubio (2011, p. 121) son:

- apoyo incondicional

- estímulo y gratificación afectiva a los logros

- vinculación afectiva

- empatía

- confianza

- capacidad para asimilar nuevas experiencias y ayudar a resolver problemas.

\section{Sentido de vida}

El pensamiento crítico en la mirada resiliente se considera un factor protector porque le permite al individuo desarrollar la capacidad de analizar las causas y responsabilidades de su adversidad (Melillo, 2004). Además, permite reflexionar sobre las ganancias, que le dan sentido a su vida.

Por otra parte, la autoestima consciente, que en resiliencia se considera como el fruto del cuidado afectivo de una persona adulta con la niñez, actúa como factor protector, porque le permite a la persona sentirse valorada y esto le provoca seguridad en sí misma.

El sentido de vida se visualiza como producto de la capacidad de analizar las situaciones adversas y poder dominar la existencia personal, la forma de planificar para darle sentido a la vida, ya que, si bien el individuo está acompañado por el otro ser, él es el único que toma la decisión de continuar; los demás le dan sentido a su vida a través del reconocimiento y ello estimula su autoestima y favorece su resiliencia (Werner y Smith, 1992).

En los siguientes testimonios puede advertirse el sentido de vida que se desarrolló a partir de haber contado con factores protectores: 
Un día decidí irme a vivir sola, ya trabajaba, ya había terminado la carrera y como me sentí con las condiciones necesarias para independizarme pues me fui. Simplemente quería dejar de confrontarme con mis padres, dejar de pedir permiso y me sentí con la confianza suficiente para irme. (MR15)

Tengo maravillosos recuerdos de mi niñez ya que en cada momento me acompañaron mis padres, recuerdo que fuimos al mar y que tenía miedo, pero mis padres me explicaron de una forma muy tierna y me dijeron que no tenía nada que temer, que iban a estar ahí. (MR10)

En ambos comentarios se advirtió que el reconocimiento da sentido a la vida del ser humano. Como somos seres sociales por naturaleza, necesitamos del otro para avanzar y seguir adelante. Algunas personas del estudiantado de posgrado en su niñez fueron acompañadas por personas que les dieron amor y esperanza. Esto le dio sentido a su vida y, por ende, sembró la semilla de su resiliencia.

\section{A manera de cierre}

La resiliencia es la capacidad del ser humano para enfrentar circunstancias adversas y salir fortalecido. Según Grotberg (2006), esta puede ser desarrollada por el individuo; sin embargo, el reto es cómo enseñar y aprender la resiliencia. Al utilizar el taller como técnica de investigación y programar como actividad que el estudiantado escribiera su historia de vida, resaltando situaciones difíciles en las que lograron salir fortalecidos se evidenció su capacidad resiliente para transformar un factor de riesgo en un factor protector. Lo anterior coincide con la aportación de Grotberg y permite afirmar que todas las personas somos resilientes.

En las historias de vida del estudiantado son evidentes las heridas en el alma, que les dieron aprendizajes para sobrevivir a la adversidad y construir su resiliencia, transformando su mirada para poder ver en lo negativo una oportunidad para ser mejor persona. Esa es la apuesta de la resiliencia.

Por lo que se evidencia la importancia de considerar, en los requisitos de ingreso al posgrado la trayectoria emocional del aspirante, ya que permitirá analizar sus actitudes frente a las adversidades $y$, por ende, su desarrollo para su futuro en la acción investigadora educativa.

Al analizar las historias de vida del estudiantado del posgrado en Pedagogía de la UNAM desde una perspectiva resiliente, se identificaron problemas en su niñez que le hirieron $y$, con el paso del tiempo, desarrollando resiliencia, han logrado transformar esos problemas en fortalezas que actualmente apoyan su toma de decisiones.

El ser humano es un investigador nato: cuando nace, empieza a desarrollar sus habilidades de investigación. Con el tiempo, en algunos casos, merman; en otros, se acentúan. ¿De qué depende? La respuesta es sencilla, pero compleja: depende del contexto en que se desarrolla. 
doi: http://dx.doi.org/10.15359/ree.22-1.7

URL: http://www.una.ac.cr/educare

CORREO: educare@una.cr

Ingresar a un posgrado significa esfuerzo y dedicación para generar tanto ideas enfocadas en identificar problemas en la educación como propuestas para su mejora, por lo que las actividades cotidianas giran en torno a la investigación y al análisis.

Descubrir factores como identificación y solución de problemas, el otro significativo o personal tutor resiliente y sentido de vida en la mirada resiliente del estudiantado de posgrado permite inferir que estos elementos fortalecen su formación en investigación educativa, ya que, según Labaree (2003), la persona investigadora educativa debe empezar por estar convencida de que, además de su experiencia, puede aprender algo valioso observando la educación desde fuera; es decir, desde la necesidad y el valor de la investigación.

Aprender a observar el problema articulando las teorías con las experiencias de vida requiere tener habilidades del pensamiento crítico y creatividad, al mismo tiempo que introspección, moral e independencia para poder analizar y emitir propuestas valiosas para la educación.

Estas habilidades evidencian el desarrollo de resiliencia en algunos estudiantes del posgrado; dado que han transformado escenarios negativos en áreas de oportunidad que les proveen confianza y seguridad, elementos necesarios, según Chivers (2006), en la formación de investigadores educativos.

Cada participante relató su propia historia, que almacena y procesa información en la memoria. Si hubo alguien que aportó amor a través de sus palabras, abrazos y caricias construyó en la niñez del estudiantado de posgrado seguridad y puntos de apoyo que lo formaron para enfrentar situaciones difíciles.

El estudiantado de posgrado, al escribir su historia, hizo un esfuerzo que lo convirtió en actor principal de la escena ya desde una mirada diferente frente a los traumas que forman ahora parte de su experiencia. A partir de los hallazgos, fue posible identificar evidencias de resiliencia, porque, al narrar sus historias, lograron identificar problemas que también solucionaron transformando algo negativo en positivo.

Al rescatar la mirada de la niñez en la persona adulta, se infiere que en la infancia se forman los pensamientos a través de lo que se siente. A medida que se va creciendo, se construyen con el pensamiento crítico. Por esto, el acercamiento a la historia de vida se torna más analítico, por lo que al vincularlo con las aportaciones de Cyrulnik (2003), Vanistendael (2006), Suárez (2004) y Melillo (2004), se identifica la construcción de la resiliencia y, en consecuencia, su desarrollo.

En la niñez el ser humano es capaz de contrarrestar la reflexión distorsionada, identificando el problema, reduciendo la ansiedad y la culpa; esto evidencia su capacidad resiliente. En cambio, en su adultez, su capacidad resiliente se muestra como sabiduría, asumir responsabilidades sin culpar a las demás personas, comprensión de sí y de otras personas (Espinosa, 2008).

\footnotetext{
16 
Asumir la responsabilidad de uno mismo o una misma es un proceso complejo que el ser humano desarrolla por el acompañamiento del otro significativo o personal tutor resiliente, cuya figura provee, gracias a su aceptación incondicional, el apoyo que lo motiva a continuar su trayectoria de vida, como el viento a las alas de una gaviota.

Por lo mismo, los procesos de interacción son imprescindibles para el desarrollo de la resiliencia, porque un individuo no puede desarrollarse más que tejiéndose con otro (Cyrulnik, 2010). En consecuencia, los hilos que entrelazan el proceso de formación de personal investigador educativo en el posgrado deben considerar la comprensión, la valoración y el acompañamiento, elementos primordiales en la tutoría que apoyan la formación integral del estudiantado.

Por lo tanto, la importancia de la interacción positiva entre el estudiantado, tutorías y profesorado es transcendental para generar ambientes que promuevan resiliencia, ya que se espera que el contexto donde se desarrolla el personal investigador educativo pueda incidir en su transformación personal y profesional. En el caso del Posgrado en Pedagogía, se evidenció que existen elementos que pueden convertirse en factores de riesgo; sin embargo, al promover el descubrimiento de la resiliencia entre el estudiantado generó expectativas positivas al darse cuenta de que, a pesar de lo vivido, han logrado ingresar y permanecer en el posgrado (Cyrulnik, 2003).

Por lo anterior, se infiere que, en el estudiantado, emerge el sentido de vida que lo sostiene, impulsa y fortalece en esos momentos en que parece que no hay camino, aparecen señales invisibles, como en el ciberespacio, que conectan al sujeto con las personas que lo aceptaron de manera incondicional, que le dieron esa sensación consciente y maravillosa que lo transforma en un ser valioso y reconocido.

Ese sentido de vida que lo acompaña y que ante las adversidades lo sostiene, que construye en los pensamientos escenarios mágicos donde aflora la capacidad para mejorar como personas y que fortalece la formación del estudiantado de posgrado como personal investigador educativo, porque le permite tomar decisiones que busquen apoyar la educación en favor de los otros seres y, por ende, también sembrar resiliencia.

\section{Referencias}

Ausubel, D. P. (1954). Theory and problems of adolescent development. United States of America: Grune y Stratton.

Chivers, G. (2006). The training of university lifelong learning professionals as researchers. Journal of European Industrial Training, 30(5), 330-348. doi: https://doi. org/10.1108/03090590610677908 
doi: http://dx.doi.org/10.15359/ree.22-1.7

URL: http://www.una.ac.cr/educare

CORREO: educare@una.cr

Cifuentes, R. M. (2011). Diseño de proyectos de investigación cualitativa. Buenos Aires: Noveduc.

Cyrulnik, B. (2003). El encantamiento del mundo. Barcelona: Gedisa. Recuperado de https://mega. nz/\#!f9BjgbxB!CNtaDzQAUgQuEZmKrgp YMI3owOstSRIfV2THnYIEZs

Cyrulnik, B. (2005). El amor que nos cura (2a ed.). Barcelona, España: Gedisa.

Cyrulnik, B. (2006). Los patitos feos. La resiliencia. Una minfancia infeliz no determina la vida. Barcelona, España: Gedisa.

Cyrulnik, B. (2008). Bajo el signo del vínculo. Una historia natural del apego. Sevilla, España: Gedisa.

Cyrulnik, B. (2010). Me acuerdo... El exilio de la infancia. Barcelona: Gedisa.

Delors, J. (Preside). (1992). La educación encierra un tesoro. Informe a la Unesco de la Comisión Internacional sobre Educación para el siglo XXI (Compendio). Madrid: Santillana Ediciones Unesco.

Erikson, E. H. (1998). O ciclo de vida completo. Porto Alegre: Artmend.

Espinosa, A. (2008). El mundo amarillo. Si crees en los sueños. Ellos se crearán. Barcelona: De bolsillo.

Fiorenza, A. (2009). Cuando el amor no basta. Consejos eficaces para resolver los problemas entre padres e hijos adolescentes. Madrid: Planeta.

García, O y Barrón, C. (2011). Un estudio sobre la trayectoria escolar de los estudiantes de doctorado en pedagogía. Perfiles Educativos, 33(131), 94-113. Recuperado de http://www. redalyc.org/articulo.oa?id=13218531007

Garmezy, N. (1991). Resiliency and vulnerability to adverse developmental outcomes associated with poverty. American Behavioral Scientist, 34(4), 416-430. doi: https://doi. org/10.1177/0002764291034004003

Ghiso, A. (1999). Acercamientos: El taller en procesos investigativos interactivos. Revista Colombiana de Trabajo Social, 13, 143-156.

Godard, F. y Cabanes, R. (1996). Uso de las historias de vida en las ciencias sociales [Series Cuadernos del CIDS, II, 1]. Bógota: Centro de Investigaciones sobre la Dinámica Social, Universidad Externado de Colombia.

Grotberg, E. H. (July, 1996). The international resilience project findings from the research and the effectiveness of Interventions. Paper presented at the Annual Convention of the International Council of Psychologists. Banff, Canada. Recuperado de http://files.eric. ed.gov/fulltext/ED419584.pdf 
Grotberg, E. H. (2006). La resiliencia en el mundo de hoy. Cómo superar las adversidades. Madrid: Gedisa.

Labaree, D. (2003). The peculiar problems of preparing educational researchers. Educational Researcher, 32(4), 13-22. doi: https://doi.org/10.3102/0013189X032004013

Laclette, J. P. (2014). Solo 16 de cada 10 mil mexicanos estudian un posgrado [Foro consultivo científico y tecnológico]. Recuperado de http://www.Foroconsultivo.org.mx

Lemaîitre, E. y Puig, G. (2005). Programa rueda. Fortaleciendo la resiliencia: Una estrategia para desarrollarlaautoestima. Santiago:USACH.Recuperado dewww.addima.org/documentos/ recursos/programa\%20rueda.pdf

Madariaga, J. M., Palma, M., Surjo, P., Villalba, C.y Arribillaga, A. (2014). La construcción social de la resiliencia. En J. M. Madariaga (Coord.), Nuevas miradas sobre la resiliencia. Ampliando ámbitos y prácticas (pp.11 - 30). España: Gedisa.

Manciaux, M. (2002). Prefacio. En S. Vanistendael y J. Lecomte. La felicidad es posible. Despertar en niños maltratados la confianza en si mismos: Construir la resiliencia. (pp. 12-12). Barcelona: Gedisa.

Martínez, A. (2013). Los beneficios de estudiar un posgrado. Viva San Carlos. Recuperado de http://www.sancarlosboard.com/post/los-beneficios-de-estudiar-un-posgrado-6382952

Mead, G. H. (1972). Espíritu, persona y sociedad. Desde el punto de vista del conductismo social. Argentina: Paidós.

Melillo, A. (2004). Resiliencia. Psicoanálisis ayeryhoy, 1. Recuperado de http://www.elpsicoanalisis. org.ar/old/numero1/resiliencia1.htm

Melillo, A. (2006). Realidad social, psicoanálisis y resiliencia. En A. Melillo, E. N. Suárez y D. Rodríguez (Comps.), Resiliencia y subjetividad. Los ciclos de la vida. Buenos Aires: Paidós.

Mella, O. (1998). Naturaleza y orientaciones teórico-metodológicas de la investigación cualitativa. Revista Científica Umbral, 1, 1-74. Recuperado de Recuperado de http://www.epiclin. unicauca.edu.co/archivos/Naturaleza\%20de\%20la\%20investigacion\%20cualitativa.pdf

Minuchin, S. y Fishman, H. C. (1984). Técnicas de terapia familiar. Barcelona: Paidós.

Morin, E. (2005). Introducción al pensamiento complejo. Barcelona: Gedisa.

Puig, G. y Rubio, J. L. (2011). Manual de resiliencia aplicada. Madrid: Gedisa.

Rousseau. S. (2012). La resiliencia. Vivir feliz a pesar de... Barcelona: Gedisa.

Rubio, J. L. y Puig, G. (2015). Tutores de resiliencia. Dame un punto de apoyo y moveré mi mundo. Barcelona: Gedisa. 
doi: http://dx.doi.org/10.15359/ree.22-1.7

URL: http://www.una.ac.cr/educare

CORREO: educare@una.cr

Rutter, M. (1979). Protective factors in children's responses to stress and disadvantage. Annals of the academic of Medicine. Singapore, 8(3), 324-338.

Rutter, M. (1985). Resilience in the face of adversity: Protective factors and resistance to psychiatric disorder. British Journal of Psychiatry, 147, 598-611. doi: https://doi.org/10.1192/ bjp. 147.6 .598

Rutter, M. y Rutter, M. (1992). Developing minds: Challenge and continuity across the life span. Londres: Penguin Books.

Suárez, E. N. (2004). Introducción: Resiliencia y subjetividad. En A. Melillo, E. N. Suárez y D. Rodríguez (Comps.), Resiliencia y subjetividad. Los ciclos de la vida. Buenos Aires: Paidós.

Suárez, E. N., De la Fara, A. M. y Márquez, C. V. (2007). Trabajo comunitario y resiliencia social. En M. M. Munist, E. N. Suárez, D. Krauskopf y T. J. Silber (Comps.), Adolescencia y resiliencia (pp. 81-108). Argentina: Paidós.

Suárez, E. N. y Krauskopf, D. (1995). El enfoque de riesgo y su aplicación a las conductas del adolescente. Una perspectiva psico-social. En M. Maddaleno, M. M. Munist, C. V. Serrano, T. J. Silber, E. N. Suárez y J. Yunes (Eds.), La salud del adolescente y del joven (pp. 183-193). Washington: OPS.

Taylor, S. J. y Bogdan, R. (2013). Introducción a los métodos cualitativos de investigación. Barcelona: Paidós.

Torres-Frías, J. (2012). Desigualdad educativa, jerarquías de poder y violencia simbólica en la formación de investigadores de la educación. Revista lberoamericana deEducación Superior, 3(8), 115-129. Recuperado de https://ries.universia.net/article/view/93/desigualdadeducativa-jerarquias-poder-violencia-simbolica-formacion-investigadores-educacion

Tuirán, R. (2012). El posgrado actual. En M. Serna y R. M. Pérez (Coords.), Logros e innovación en el posgrado (pp. 15-18). Morelia, México: COMEPO. Recuperado de http://www.comepo.org. mx/images/publicaciones/logros-e-innovacion-en-el-posgrado.pdf

Vanistendael, S. (2006). La resiliencia: Desde una inspiración hacia cambios prácticos. En Mapfre (Ed.), $2^{\circ}$ Congreso internacional de los trastornos del comportamiento en niños y adolescentes (pp. 1-13). Madrid, España. Recuperado de https://www.obelen.es/upload/262D.pdf

Vanistendael. (2014). Resiliencia: El reto del cambio de mirada. En J. M. Madariaga (Coord.), Nuevas miradas sobre resiliencia. Ampliando ámbitos y prácticas (pp. 53-67). Madrid: Gedisa.

Vanistendael, S. y Lecomte, J. (2002). La felicidad es posible. Despertar en los niños maltratados la confianza en sí mismos: Construir la resiliencia. Madrid: Gedisa. 
Vélez, O. L. y Galeano, M. E. (2000). Investigación cualitativa. Estado del arte. Colombia: Editorial Universidad de Antioquia.

Watzlawick, P. (1976). ¿Es real la realidad? Confusión, desinformación, comunicación. Barcelona: Herder.

Werner, E. E. (1989). High-risk children in young adulthood: A longitudinal study from birth to 32 years". The American Journal of Orthopsychiatry, 59(1), 72-81. doi: https://doi. org/10.1111/j.1939-0025.1989.tb01636.x

Werner, E. E. y Smith, R. S. (1982). Vulnerable but invincible. A longitudinal study of resilient children and youth. New York: Mc Graw Hill.

Werner, E. E. y Smith, R. S. (1992). Overcoming the odds: High risk children from birth to adulthood. Ithaca, NY: Cornell University Press. 